\title{
A biology-based approach for quantitative structure-activity relationships (QSARs) in ecotoxicity
}

\author{
Tjalling Jager - Sebastiaan A. L. M. Kooijman
}

Accepted: 30 September 2008/Published online: 19 October 2008

(c) The Author(s) 2008. This article is published with open access at Springerlink.com

\begin{abstract}
Quantitative structure-activity relationships (QSARs) for ecotoxicity can be used to fill data gaps and limit toxicity testing on animals. QSAR development may additionally reveal mechanistic information based on observed patterns in the data. However, the use of descriptive summary statistics for toxicity, such as the 4-day LC50 for fish, introduces bias and ignores valuable kinetic information in the data. Biology-based methods use all of the toxicity data in time to derive time-independent and unbiased parameter estimates. Such an approach offers whole new opportunities for mechanism-based QSAR development. In this paper, we apply the hazard model from DEBtox to analyse survival data for fathead minnows (Pimephales promelas). Different modes of action resulted in different patterns in the parameter estimates, and therefore, the toxicity data by themselves reveal insight into the actual mechanism of toxic action.
\end{abstract}

Keywords QSAR · DEBtox · Survival ·

Biology-based modelling · Toxicity

\section{Introduction}

Lack of toxicity data is a serious limitation for environmental risk assessment in a regulatory context (Bradbury et al. 2004). Quantitative structure-activity relationships (QSARs) may be applied to fill these data gaps and limit testing on animals. The standard approach in developing

T. Jager $(\bowtie) \cdot$ S. A. L. M. Kooijman

FALW/Department of Theoretical Biology, Vrije Universiteit

Amsterdam, De Boelelaan 1085, 1081 HV Amsterdam,

The Netherlands

e-mail: tjalling@bio.vu.nl
QSARs for toxicity is to collect toxicity values for one species for a group of chemicals (usually sharing a presumed mechanism of action), and attempt to find one or a few molecular descriptors that, in some form of regression, provide an adequate description. This approach has been very popular over the last decades, and has yielded a variety of QSAR equations (see e.g., Bradbury 1995; Schultz et al. 2003). However, progress in this field has been limited to developing equations for new species, new groups of toxicants, and using other descriptors. The toxicity values themselves are treated as given facts, rather like they were analytical measurements of toxicity. We will argue here that the currently used summary statistics (e.g., LC50) are a poor representation of the toxicity of chemicals, which introduces bias, obscures patterns and hampers the predictive value of QSARs. The development of mechanistically meaningful QSARs requires critical scrutiny of the methods to derive summary statistics, and consideration of biology-based alternatives.

The measure of toxicity that is used to develop QSARs is almost always the concentration causing a specific level of effect (e.g., 10 or $50 \%$ ) on organism response after a standardised exposure time. For example, acute toxicity to fish is presented as the 4-day LC50. However, it has long been known that LC50s decrease in time in a more or less predictable manner until they reach a stable level, i.e., the incipient LC50 (Sprague 1969). The time needed to reach this level depends, among other things, on the toxicokinetics, which is affected by properties of the compound (e.g., hydrophobicity and mechanism of toxicity) and properties of the species (e.g., lipid content and size). For large fish or very hydrophobic compounds, 4 days will not be sufficient to observe the incipient LC50. Additionally, compounds that owe their toxicity to a slow formation of toxic metabolites may also require more than 4 days to 
reach the incipient level. As a result, the 4-day LC50 values for such compounds will be higher than the incipient levels, thus causing bias in QSAR regressions. Ironically, the standardised exposure time is not facilitating but actually hampering the comparison of LC50 values between chemicals and between species.

An additional limitation of focussing on the LC50 as a measure of toxicity is that a wealth of kinetic information in the data is thereby ignored. The standard test protocols for fish and Daphnia prescribe that survival is scored every day. However, this information is not used to derive LC50s or in QSAR development but does contain valuable information on the kinetic and dynamic processes that govern toxicity. To extract all relevant information from toxicity test results requires biology-based methods (OECD 2006), such as DEBtox (Bedaux and Kooijman 1994; Jager et al. 2006). These methods make use of all of the observations over the entire exposure time to extract parameter values that are independent of test duration. Because the resulting parameters represent actual processes in the organism, it is likely that they are better described by molecular properties, and that these relationships contain more meaningful mechanistic information. Additionally, the parameters of biology-based models are expected to co-vary in specific ways (Kooijman et al. 2007), which offers unique opportunities for the development of predictive QSARs.

In this paper, we explore the potential of biology-based modelling in QSAR development. Actual validated QSARs will not be presented, but we will demonstrate how these methods can lead to a different approach toward QSARs. In this paper, we will limit ourselves to the endpoint mortality, and present an analysis of toxicity data for fathead minnows (Pimephales promelas). An extensive discussion of alternative concepts for biology-based analysis of survival data has been presented by Ashauer and Brown (2008); we focus on one particular method, the hazard model as applied in DEBtox, which is able to work with data as provided by the use of standard test protocols (OECD 2006).

\section{Theory}

\section{Biology-based modelling}

In biology-based modelling, we attempt to explain toxic effects as a function of exposure concentration and time, from a set of consistent assumptions about the underlying processes (Jager et al. 2006; OECD 2006). This requires the explicit consideration of toxicokinetics because chemicals need to be taken up into the organism's body to produce an effect. Usually, we do not have access to internal concentrations in toxicity experiments. The toxicity pattern in time does not hold information on the absolute body residues, but does provide insight into the time needed to achieve a steady state, and thus on the elimination rate. To accommodate this limited information content, we can use a scaled internal concentration, defined as the true (but unknown) body residue divided by the (also unknown) bioconcentration factor (Bedaux and Kooijman 1994). The scaled internal concentration is directly proportional to the real body residues, but with the unit of the external concentration. If we assume a one-compartment model with first-order kinetics, we obtain the following expression for the scaled internal concentration $c_{\mathrm{V}}$ :

$\frac{\mathrm{d}}{\mathrm{d} t} c_{\mathrm{V}}=k_{\mathrm{e}}\left(c_{\mathrm{e}}-c_{\mathrm{V}}\right)$

where $c_{\mathrm{e}}$ is the external concentration in the medium. The scaled internal concentration can subsequently be linked to the toxic effects (as will be done in Eq. 2). In this way, by fitting the toxicity data in time, we can estimate the elimination rate $\left(k_{\mathrm{e}}\right)$ from the data. Of course, when actual body residues are measured, or when reliable $\mathrm{BCF}$ data are available, an unscaled one-compartment model can be used instead of Eq. 1. It must be stressed that the elimination rate estimated from toxicity data will represent the toxicokinetics (of the relevant metabolite) at the relevant target site, and does not necessarily equal the whole-body elimination rate as derived from bioaccumulation studies.

Before we can analyse survival data, we have to make assumptions regarding the mechanism of death (for a discussion of mortality concepts, see Kooijman 1996; Newman and McCloskey 2000; Zhao and Newman 2007). The hazard model of DEBtox (Bedaux and Kooijman 1994) is based on the assumption that mortality is best treated as a chance process: the internal concentration of the toxicant increases the probability to die. The statistical technique to deal with chance events in time is hazard modelling; also known as failure-time analysis in industrial applications (Muenchow 1986). Calculation is performed through the hazard rate; the hazard rate multiplied by a short time interval gives the probability to die in that interval, provided the organism is alive at the beginning of that interval. The hazard rate is linked to the scaled tissue concentration (Eq. 1), which changes in time. For the relationship between these two properties, we take a simple linear relationship with a threshold. This threshold $\left(c_{0}\right)$ is a true no-effect concentration or NEC (in contrast to the NOEC; Kooijman 1996): exposure below this level does not lead to mortality (other than background mortality), even after prolonged exposure. Above the NEC, the hazard rate is proportional to the part of the scaled concentration that exceeds the threshold. The proportionality constant is called the killing rate $\left(b_{\dot{\dagger}}\right)$. This 
yields the following equation for the hazard rate due to the toxicant $\left(h_{\mathrm{c}}\right)$ :

$h_{\mathrm{c}}=b_{\dagger} \max \left(c_{\mathrm{V}}-c_{0}, 0\right)$

where $c_{\mathrm{V}}$ is given by Eq. 1 . Because $c_{\mathrm{V}}$ is in external concentration units, $c_{0}$ also is expressed as an external concentration, and $b_{\dagger}$ is expressed in per external concentration per time. The NEC is thus the external concentration that will not lead to exceedance of the unknown internal threshold, even after long exposure.

Because the internal concentration is a function of exposure time, the hazard rate will also change in time. The fraction survival $(q)$ in time can be calculated by integrating the hazard rate over time:

$q(c, t)=\exp \left(-\int_{0}^{t}\left(h_{\mathrm{c}}(c, \tau)+h_{0}\right) \mathrm{d} \tau\right)$

where the blank mortality rate $\left(h_{0}\right)$ is added to the hazard rate due to the toxicant. For standard acute tests we can assume a constant (low) background hazard rate and a constant exposure concentration. Therefore, these three equations can be combined and solved analytically, which speeds up the calculations (Bedaux and Kooijman 1994). The analysis of survival data thus requires three toxicological parameters: a NEC, killing rate, elimination rate, and a single organism parameter (the background hazard rate) that is independent of the toxicant. These parameters are independent of exposure time, at least within the duration of the experiment.

\section{Example calculation}

To illustrate how the hazard model deals with survival data sets, we provide an example for a 4-day acute toxicity study for hexachlorobutadiene in fathead minnows (data from Geiger et al. 1985). A standard descriptive analysis yields an LC50 at the end of the test of $0.38 \mu \mathrm{M}$ (as reported by the original authors). The test contains daily observations on mortality, and the hazard model uses all data together in one integrated analysis (Fig. 1). The resulting NEC is $0.13 \mu \mathrm{M}$ (95\% confidence interval 0.0910.16 ), the elimination rate $0.012 \mathrm{~h}^{-1}$, and the killing rate $0.20 \mu \mathrm{M}^{-1} \mathrm{~h}^{-1}$. The background hazard rate is close to zero. Note that the model analysis requires four parameters, irrespective of the number of observation times to describe the entire concentration-time-response surface, whereas a standard regression analysis would require two or three parameters per time point.

The right panel of Fig. 1 shows the lines of equal effect over time. Clearly, LCx values decrease over time, which reflects toxicokinetics (the time needed to establish steady state, through $k_{\mathrm{e}}$ ) and toxicodynamics (the increase of the probability to die with increasing body residues, through $\left.b_{\dot{\dagger}}\right)$. The iso-effect lines eventually converge at the NEC for long exposure times. This implies that the concentrationresponse curve (which is not shown) gets steeper in time, until it is nearly vertical and the LC0 will approach the LC50. The NEC is therefore numerically identical to the incipient LC50. For this particular compound, the LC50 has not yet reached the NEC at the end of the test. In other words, the LC50 would have decreased further had the test been continued for longer than the standard 4 days.

It must be stressed that the time needed to achieve the incipient LC50 is not fully determined by the whole-body elimination rate, and therefore, hydrophobicity of the compound is a limited indicator of optimal test duration. Firstly, in the hazard model, the killing rate determines the time to reach the incipient LC50 together with the elimination rate. A low killing rate implies that more time is needed to achieve the incipient LC50 than to reach steady state body residues. The second limitation of hydrophobicity as a proxy for optimal test duration lies in the applicability of the one-compartment model. Even though this model often works well in practise, it is certainly possible that the relevant kinetics at the target site is better described by a multi-compartmental approach, or a different kind of kinetics.

\section{Theoretical considerations on parameter values}

Unlike descriptive regression models, the parameters of biology-based methods have a physiological meaning. This means that the parameters of biology-based models cannot vary independently, and in fact, we can expect a priori to see strong relationships between the parameters for chemicals that share a mechanism of toxicity (Kooijman et al. 2007). To illustrate these patterns, we will start with chemicals exhibiting non-polar narcosis or "baseline toxicity". Even though the exact mechanisms behind this mode of action are unclear, it appears that the target sites are the cell membranes throughout the body (Escher and Hermens 2002). For the amount of effect, it does not seem to matter whether we have a molecule of compound A or B in the cell membrane. Therefore, the relationship between the level of target occupation (i.e., the number of molecules in the membranes) and the hazard rate is expected to be compound independent. This implies that the NEC and killing rate of all narcotic compounds will be the same when these parameters are expressed on internal molar concentrations. However, we used the scaled internal concentration (Eq. 1) instead of the actual internal concentration, which differs by a factor that equals the bioconcentration factor. Thus, different narcotic chemicals differ in NEC and killing rate, not because they are 
Fig. 1 Hazard model fit for survival of fathead minnow (Pimephales promelas) exposed to hexachlorobutadiene. The right panel shows the iso-effect lines for 0,10 and $50 \%$ effects
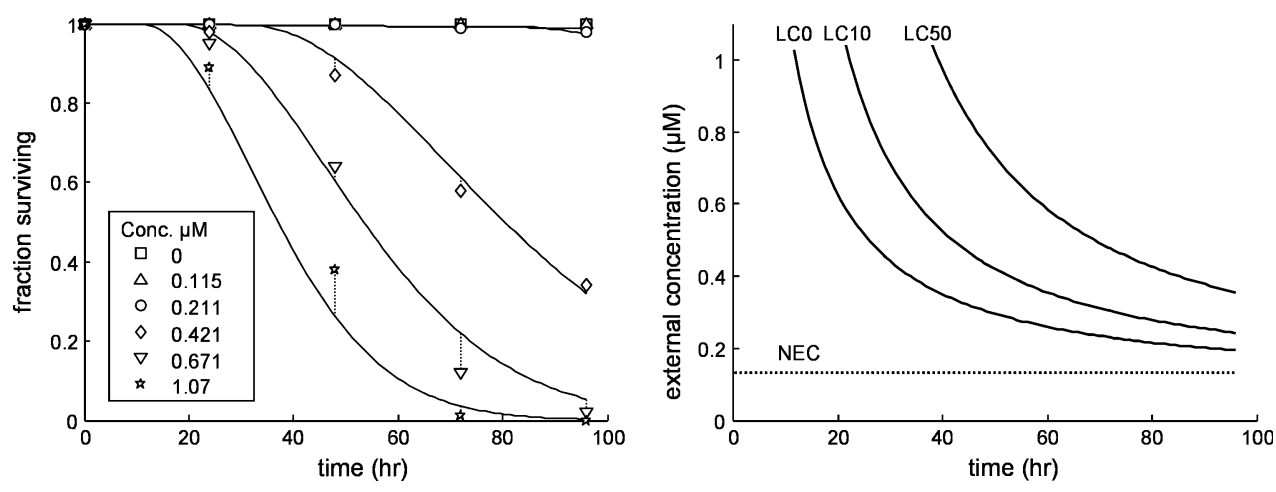

inherently more or less toxic, but because they differ in their degree of bioconcentration, and thus the efficiency with which they are taken up and reach the target. The NECs and killing rates for different narcotic compounds will therefore be inversely proportional; plotting NECs versus killing rates on $\log -\log$ scale should yield a line with a slope of exactly -1 . Because hydrophobicity drives the concentration in the cell membrane, the NEC and killing rate should show a strong correlation to $K_{\text {ow }}$ (as a proxy for membrane lipids). Such strong correlations between hydrophobicity and these model parameters were previously observed for Daphnia magna exposed to a series of alkylphenols (Gerritsen et al. 1998), which are expected to be narcotic (Russom et al. 1997).

For other mechanisms of toxicity, we expect to see the same inverse proportionality between NEC and killing rate, when plotting compounds with the same toxicity mechanism; a slope of exactly -1 on log-log scale. Following the same argument as for narcosis, it should not matter whether, for example, acetyl cholinesterase is inhibited by organophosphate A or B. At the level of the target, the NEC and killing rate should be the same for all inhibitors (see Jager and Kooijman 2005). However, the factor between target occupation and scaled internal concentration now includes the interaction efficiency with the target, in addition to the bioconcentration factor. A correlation of the NEC or killing rate with $K_{\mathrm{ow}}$ is therefore not selfevident anymore for such specific mechanisms of action.

In contrast to the slopes, the intercepts of the relationships between NEC and killing rate will differ between the various mechanisms of toxicity. As such, this provides an excellent opportunity to classify chemicals, or to test current mode of action classifications. Deviations from this strict inverse proportionality between NEC and killing rate may occur in practise, due to experimental error and biological variation, but also because the mechanism of effects may be more complicated than assumed (e.g., include nonlinear biotransformation steps). Additionally, compounds may deviate from strict proportionality because they do not actually have the same mechanism of action (misclassification), or a compound may affect more than one target in an organism.

Strong relations between the elimination rate and the NEC or killing rate are not expected, as the elimination rate is to some extent independent of the actual mechanism of toxicity. In an earlier paper (Kooijman et al. 2004), we discussed the relationship between hydrophobicity and elimination rates. We expected either that the elimination rates scale with the square root of $K_{\mathrm{ow}}$ (leading to a linear relation on $\log -\log$ scale, with a slope of -0.5 ), follow a two-stage relationship (constant at low $K_{\mathrm{ow}}$, slope of -1 at high $K_{\mathrm{ow}}$ ), or a mixed form of these two extremes. It should be stressed that these relationships with hydrophobicity are expected for the elimination rate of the whole-body residue, but that mortality is determined by the kinetics at the relevant target site. Especially for chemicals with a nonnarcotic mode of action, the toxicity-based elimination rate ( $k_{\mathrm{e}}$ of Eq. 1) results from the one-compartment approximation of a more complex behaviour, and the value of the rate constant can differ from measurements based on whole-body concentrations, or values predicted on the basis of hydrophobicity (e.g., for organophosphates; Jager and Kooijman 2005).

\section{Methods}

Data selection

The use of DEBtox, and other biology-based methods, requires the original raw data from toxicity experiments (the number of surviving organisms over time). Unfortunately, ecotoxicological databases only store simple summary statistics such as LC $x$ values; the underlying raw data have been lost or are difficult to trace. One of the exceptions is the work of the Center for Lake Superior Environmental Studies (Brooke et al. 1984; Geiger et al. 1985, 1986, 1988, 1990), describing the test results from 4-day acute tests with 
fathead minnow. Data from these reports will therefore serve as a demonstration in this paper. The tests have been conducted with juvenile minnows (approx. $2 \mathrm{~cm}$ in length) at constant exposure (flow-through, generally five doses and a blank, exposure concentrations measured at several time points), and at a water temperature around $25^{\circ} \mathrm{C}$. The experimental setup comprised a variable number of observations in time (generally 3-8), and variable number of animals per dose group (generally 10-100). We used the average measured exposure concentrations, corrected for recovery, and expressed in $\mathrm{mM}$. Data for the following classes of compounds were analysed: (halogenated) aliphatic hydrocarbons (class 1 and 2), ethers (class 3), alcohols (class 4), aldehydes (class 5), ketones (class 6) and benzenes (class 13). Chemical properties $\left(\log K_{\text {ow }}\right.$ and molecular weight) were taken from EPI Suite 3.12. For $\log K_{\text {ow }}$, estimated values were used to provide consistency as measured values are not available for all compounds. For the most likely mode of action, the classification of Russom et al. (1997) was taken. The most common mode of action for our selected classes were narcosis 1 and electrophile/ pro-electrophile reactivity. Only those compounds for which it is quite certain that they are indeed non-polar narcotics or reactives are included (level of confidence A or B, see Russom et al. 1997).

To illustrate inter-species generalities, the $K_{\mathrm{ow}}$-relationships of the hazard model's parameters derived by Gerritsen et al. (1998) for Daphnia magna exposed to alkyl phenols will also be included, together with the minnow data for narcotics.

\section{Fitting the DEBtox hazard model}

The hazard model (Eqs. 1-3) was fitted to the raw survival data, yielding estimates for all four parameters: NEC, killing rate, elimination rate and background hazard rate. Robust confidence intervals were generated using profile likelihoods (Meeker and Escobar 1995). All calculations were performed with Matlab version 7.3. The model procedure was not in all cases able to accurately identify all four parameter values from the data, which is reflected in the width of the confidence intervals. When the entire $95 \%$ confidence interval spans less than one order of magnitude, we considered the estimate to be of "sufficient confidence" and indicated these values in the figures with a filled symbol. For elimination rates, a slightly different quality criterion was used. In some cases, a very high elimination rate fits the data best, which implies nearly instantaneous steady state, prohibiting an accurate estimate for the elimination rate. For plotting convenience, these values are plotted at $100 \mathrm{~h}^{-1}$ in the graphs, and are considered "accurate" only when the $95 \%$ confidence interval does not extend below $30 \mathrm{~h}^{-1}$.

\section{Results and discussion}

Non-polar narcosis

First, we will focus on the chemicals from the selected chemical classes that are classified as narcosis 1. In Fig. 2, the hazard model parameters as estimated from the survival data are plotted against $\log K_{\mathrm{ow}}$. The blank hazard rate does not show any relationship with hydrophobicity, as is to be expected. Interestingly, this parameter is either estimated at a very low level (effectively zero), or roughly between $10^{-4}$ and $10^{-3} \mathrm{~h}^{-1}$, equivalent to $1-9 \%$ mortality over 4 days. The NEC is estimated with high accuracy for almost all data sets (in $95 \%$ of the cases, the confidence interval spans $<0.3$ units on log scale), even though the data sets vary considerably in the number of observations in time and number of fish per dose. The NEC decreases with hydrophobicity, as expected, showing a good correlation with $\log K_{\mathrm{ow}}$, with a slope and intercept that are comparable to QSARs for the LC50 of narcotic chemicals (Veith et al. 1983). This implies that the test duration of 4 days is sufficient to observe the incipient LC50 for these compounds in this species and this size class. As illustrated in Fig. 1, the incipient LC50 will be numerically equivalent to the NEC. Indeed, a comparison between the NEC and the 4-day LC50, as reported in the original publications, shows that their values are almost identical for this data set, with a maximum deviation of a factor of 1.7. Theoretically, the similarity between the 4-day LC50 and the NEC would break down for very hydrophobic compounds. Unfortunately, this prediction could not be verified; very hydrophobic chemicals were tested but did not reveal toxicity at the solubility limit after this short test duration.

Also as expected, the killing rate shows a general increase with $K_{\text {ow }}$ but the pattern is less clear than for the NEC. This is partly caused by the fact that, in contrast to the NEC, many of the data sets do not allow for an accurate identification of this parameter. When only the points of sufficient confidence (95\% confidence interval spanning less than a factor of 10) are considered, the relationship is much clearer. The elimination rate is also more difficult to accurately identify from the data than the NEC. In several cases, the kinetics seem to be very fast (these points are plotted at $100 \mathrm{~h}^{-1}$ ), although only a few of these points are considered sufficiently accurate. In general, these elimination rates are quite high, when compared to a general QSAR for elimination in fish (Spacie and Hamelink 1982), likely because that regression was based on larger individuals ( $0.6 \mathrm{~g}$ guppies and $9 \mathrm{~g}$ trout, versus $0.1 \mathrm{~g}$ fathead minnows in the toxicity tests).

It is difficult to distinguish a clear relationship with $K_{\text {ow }}$ for the elimination rate, also because data for very hydrophobic compounds $\left(\log K_{\mathrm{ow}}>4\right)$ are scarce. Contrary to 
Fig. 2 Hazard model parameters for non-polar narcotics in fathead minnows plotted against hydrophobicity. Filled symbols represent data points with sufficient accuracy (see text). Lines in top plots represent regression equations on the filled data points. Parameter estimates for Daphnia magna from Gerritsen et al. (1998). For the elimination rate, model lines represent the toxicokinetic model of Sijm and Van der Linde (1995), parameterised for $P$. promelas
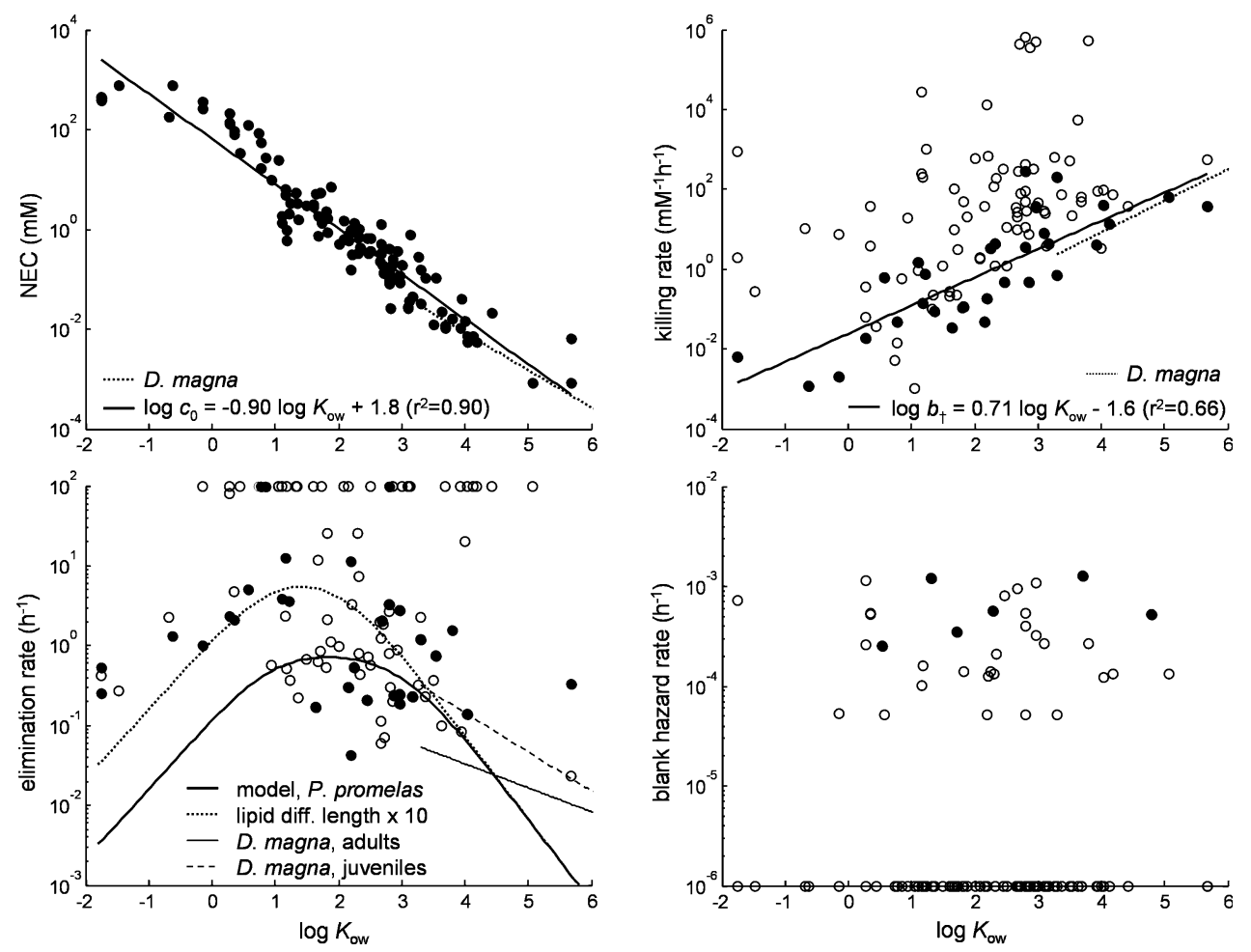

our initial expectations (Kooijman et al. 2004), the overall pattern suggests a sort of maximum elimination rate around a $\log K_{\text {ow }}$ of 1 . This pattern is, however, consistent with the toxicokinetics model of Sijm and Van der Linde (1995), which includes a detail that is specifically relevant to very hydrophilic compounds. At low hydrophobicity, the wholebody bioconcentration factor becomes constant, as it is dominated by the behaviour of the non-lipid fraction (mainly water) in the fish. The membrane-water partition coefficient, however, still decreases with decreasing $K_{\text {ow }}$. The net result is that the elimination rate will decrease when $K_{\text {ow }}$ decreases below a $\log K_{\text {ow }}$ of around 1 or 2 . Their toxicokinetics model is consistent with the overall pattern in the elimination rates, especially when decreasing the lipid diffusion length by a factor of 10 (the correct value of this parameter is not clear). For the model predictions in Fig. 2, the fish parameters of the Sijm and van der Linde model were set to representative values for these fathead minnows $(0.1 \mathrm{~g}$ body weight, $10 \%$ lipid content).

It is difficult to base firm conclusions on this analysis; it is apparently difficult to obtain reliable estimates for the elimination rate from these survival data alone, and the resulting values reveal considerable scatter (Fig. 2). It is possible that hydrophobicity is not a very good descriptor of the elimination rates of fish for this rather diverse group of compounds. On the other hand, we should also consider the possible effects of misclassification (not all of these compounds may behave purely narcotic) and metabolism. Nevertheless, in our opinion, the data in Fig. 2 are still consistent with the idea that the kinetics of the whole-body residue may be a good measure for the kinetics at the target site. However, combined toxicity and bioaccumulation studies are needed to settle this question.

It should be noted that the NEC does not show the same deviating response at low $K_{\mathrm{ow}}$ values as the elimination rate, because the NEC is not determined by the BCF but purely by the membrane-water partition coefficient, which for non-polar compounds is generally close to the $K_{\text {ow }}$ (Escher and Hermens 2002). This confirms that the target for non-polar narcotics is related to the membranes and not the whole-body tissue concentration, and illustrates how the toxicity data themselves can provide insight into the underlying mechanism.

The parameter estimates for the NEC and killing rate in D. magna, from Gerritsen et al. (1998), are well in line with our data for fathead minnows. This indicates that these parameters for narcotic compounds may be representative for a wide range of species, which is also supported by the very small sensitivity differences between species for acute narcotic effects, as observed by Jager et al. (2007). The toxicity-based elimination rates for Daphnia do not appear to differ much from those of the minnows, although the fish data in this $K_{\mathrm{ow}}$ range are rather poor. Based on their large body surface area relative to their volume, one might expect Daphnia to show much larger elimination rates. However, the large gill surface of the fish may make these two species more comparable in toxicokinetics than often assumed. 


\section{Reactive chemicals}

Figure 3 shows the estimated parameters for compounds classified as electrophile/pro-electrophile reactive. As observed for narcotic compounds, the blank hazard rate is either estimated effectively at zero, or between $10^{-3}$ and $10^{-4} \mathrm{~h}^{-1}$. Because background mortality should indeed be independent of the mechanism of toxicity, this provides confidence in the general behaviour of the model. The NEC is generally estimated with high accuracy from the survival data (in $75 \%$ of the cases, the confidence interval spans $<0.25 \log$ unit). For the reactive compounds, the trends with $\log K_{\text {ow }}$ are different than for the narcotics: hydrophobicity is a rather poor descriptor of the NEC and killing rate, and the slope of the relationships is shallower than for narcotics. This poor performance of hydrophobicity is well established, and other descriptors have been proposed (see e.g., Hermens 1990).

The elimination rate estimated from the survival data shows no relationship with $K_{\text {ow }}$; all compounds have a rather similar apparent elimination rate, which is generally lower than for the narcotic compounds of Fig. 2. For narcotics, we assumed that the elimination rates reflected the kinetics of the whole-body residues. There is no reason to believe that reactives have very different whole-body elimination kinetics than narcotics, and therefore the estimates in Fig. 3 indicate that it is not uptake in the organism that is the rate-limiting step in the toxicokinetics. The constancy of the rate constants points at a common kinetic mechanism for all compounds. Reactive chemicals act by direct chemical reaction to biological macromolecules, which can be considered "irreversible binding" (Verhaar et al. 1999). The relevant toxicokinetics will thus be more complex than the simple one-compartment model of Eq. 1, and the apparent elimination rate, as derived from the hazard model, is likely an approximation of the rate-limiting step in this mechanism. This rate-limiting step may very well be the turn-over rate of the target molecules (i.e., the replacement of irreversibly damaged macromolecules), which should be independent of the chemical's properties. We made a similar suggestion for the action of acetylcholinesterase inhibitors (Jager and Kooijman 2005).

In contrast to narcotics, the 4-day LC50 for reactive compounds is in many cases higher than the NEC (on average a factor of 1.4 , with a maximum of 3.1 , excluding the points of less confidence). This leads to the conclusion that 4 days may not be enough to achieve the incipient LC50 for reactives, independent of their $K_{\text {ow }}$.

\section{Relationships between parameters}

We predicted that the killing rate and the NEC would show a strict inverse proportionality for compounds with the same mechanism of toxicity. In Fig. 4, the killing rates are plotted against the NEC values (only values selected as accurate). For reactive compounds, the relationship between these two parameters is much stronger than for both parameters to hydrophobicity (Fig. 3). In Figs. 2 and 3, deviations from a
Fig. 3 Hazard model parameters for reactive chemicals in fathead minnows plotted against hydrophobicity. Filled symbols represent data points with sufficient accuracy (see text). Solid lines represent regression equations on the filled data points; dotted lines are the relations for narcotic compounds (see Fig. 1) for comparison

slope of -1 , and scatter in the regression, also results from the fact that hydrophobicity is a less than perfect descriptor for the relevant concentration at the target. This source of uncertainty is entirely removed by plotting the killing rate versus the NEC (Fig. 4). This is why we can force a slope of -1 on the regression; any other slope value would not make physiological sense.

The parameter estimates are clearly consistent with a slope of -1 on $\log -\log$ scale for each mode of action; there is an inverse proportionality between both parameters. The intercepts for both modes of action are significantly different; the confidence intervals of the intercepts do not overlap. Nevertheless, considerable scatter remains, making it difficult to identify a compound as reactive or narcotic based on these model parameters. Part of this scatter results from the fact that the killing rate is often not accurately identifiable from the survival data. However, it is also possible that chemicals have been misclassified, as classification is usually not based on strong biochemical evidence. Furthermore, many of these compounds may be metabolised to some extent by the fish, possibly leading to deviations from a strict proportionality. It is interesting to observe that the relationship between NEC and killing rate is stronger for reactives than narcotics. Perhaps, the narcotic mode of action is not as homogeneous as previously assumed; perhaps it does matter for the effect whether compound $\mathrm{A}$ or $\mathrm{B}$ is dissolved in the cell membrane, contrary to previous assumptions.

The parameter estimates for alkylphenols in Daphnia are not plotted in this figure, but also show a reasonably good correlation $\left(r^{2}=0.60\right)$. The regression line for this species lies in between the lines for narcotic and reactive compounds in the minnows.

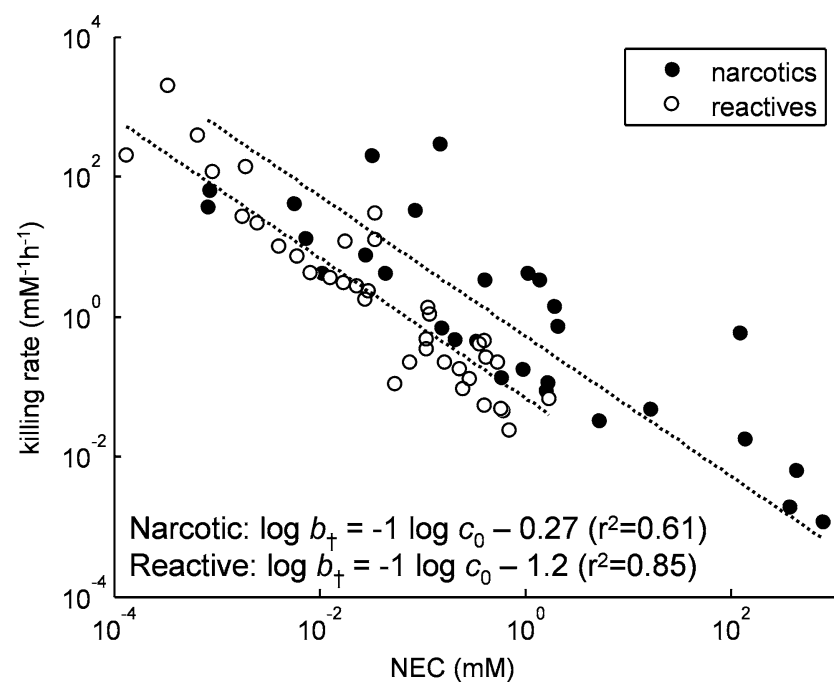

Fig. 4 Killing rate versus the NEC for narcotic and reactive compounds. Only data points of sufficient accuracy (see text) are shown. The linear regressions have a forced slope of -1
Plotting the elimination rate versus the NEC or killing rate does not lead to clear patterns. This could also not be expected, because the elimination rate is to some extent independent from the NEC and killing rate. This is illustrated by the almost constant elimination rates for reactive compounds (Fig. 3), and the deviating behaviour for narcotics for very hydrophilic compounds (Fig. 2).

\section{Outlook}

In the development of QSARs, the toxicity data are usually taken for granted. However, concepts like the 4-day LC50 make rather poor summary statistics for toxicity, which is inherent to descriptive dose-response analysis. Using a biology-based approach such as DEBtox provides a more robust and more informative view of the toxicity data. In this paper, we demonstrated the potential of this method by analysing survival data for fathead minnows. It should be noted that these bioassays have not been designed to accommodate biology-based data analysis. Nevertheless, the DEBtox hazard model provided a good fit to the experimental data in almost all cases, and the NEC could be estimated with high accuracy. This supports the application of the NEC as a robust summary statistic for risk assessment purposes (Kooijman 1996; Kooijman et al. 1996). In contrast, the kinetic parameters (killing rate and elimination rate) were more difficult to estimate accurately from these data. More observations in time would be helpful to successfully extract these parameters.

Several general conclusions could be drawn from the fathead minnow data. Firstly, the simple one-compartment model of Eq. 1 is limited for the analysis of toxicity data; the relevant toxicokinetics for mortality is not necessarily the kinetics of the whole-body residues. For narcotic chemicals, the elimination rates from the survival data could be consistent with predictions for the whole-body residue. However, for reactive compounds, the relevant kinetics are much slower and independent of hydrophobicity. In such cases, the toxicity-based elimination rate $\left(k_{\mathrm{e}}\right)$ is a one-compartment approximation of more complex kinetics, and its value can provide insight into the toxic mechanism and help to classify compounds. On a related note, this finding also implies that the optimal exposure duration is not fully determined by the hydrophobicity of the chemical. For narcotic compounds, 4 days exposure in juvenile fathead minnows is generally sufficient to achieve the incipient LC50 (at least up to a $\log K_{\mathrm{ow}}$ of 4). However, even hydrophilic reactive compounds may require more time.

Because biology-based approaches focus on the underlying mechanisms of toxicity, its parameters cannot vary independently. We have strong theoretical reasons to, a 
priori, expect certain relationships between the model parameters. For instance, the NEC and killing rate should be inversely proportional for compounds with the same mechanism of toxicity. This pattern is generally confirmed by the data presented here, which not only supports the classification of these compounds into rather homogeneous classes, but also lends credibility to the use of the NEC and killing rate as descriptors of toxicity. However, even though the data in Fig. 4 clearly indicate a slope of -1 , the scatter is considerable. Part of this variation is undoubtedly caused by experimental noise, but metabolism may have significantly contributed. It would be interesting to confirm these findings in test species with a lower metabolic capacity (e.g., Daphnia), or in the presence of a metabolic inhibitor. However, the limited data available for alkylphenols in Daphnia show a comparable degree of scatter (data not shown).

In our opinion, biology-based approaches for toxicity QSARs offer valuable possibilities, not only in the extraction of information on toxicity mechanisms, but also in their application in a regulatory setting. Firstly, the presented hazard model does not suffer from the bias inherent to the use of the 4-day LC50, as explained in the example calculation. Furthermore, because the model parameters have a physiological interpretation, they provide a better starting point for extrapolation to other compounds, other body sizes, other temperatures, time-varying exposure, etc. (Jager et al. 2006). Although we only focussed on lethal effects, a similar approach can be followed for sub-lethal endpoints such as growth and reproduction (which would be far more relevant for regulatory purposes). For such endpoints, an incipient NOEC or EC $x$ does not exist, leaving even more room for bias in QSARs due to the time-dependence of the effects (Alda Álvarez et al. 2006; Jager et al. 2006). However, for biology-based methods to be applied, the original raw data from the experiments are required, which are hardly ever reported or stored in (publicly available) databases. We therefore strongly recommend that the raw data are included in databases for future re-analysis. Furthermore, standard test protocols can be optimised for analysis with biologybased methods (Jager et al. 2006).

\begin{abstract}
Acknowledgments This study was financially supported by the European Chemicals Bureau of the European Commission, Ispra, Italy, through contract CCR.IHCP.C432545.X0. Furthermore, we acknowledge the financial support of the European Union through the integrated projects NoMiracle (Contract 003956, http://nomiracle. jrc.it) and ModelKey (Contract 511237-GOCE, http://www. modelkey.ufz.de).
\end{abstract}

Open Access This article is distributed under the terms of the Creative Commons Attribution Noncommercial License which permits any noncommercial use, distribution, and reproduction in any medium, provided the original author(s) and source are credited.

\section{References}

Alda Álvarez O, Jager T, Nuñez Coloa B, Kammenga JE (2006) Temporal dynamics of effect concentrations. Environ Sci Technol 40:2478-2484. doi:10.1021/es052260s

Ashauer R, Brown CD (2008) Toxicodynamic assumptions in ecotoxicological hazard models. Environ Toxicol Chem 27:1817-1821. doi:10.1897/07-642.1

Bedaux JJM, Kooijman SALM (1994) Statistical analysis of bioassays based on hazard modelling. Environ Ecol Stat 1:303-314. doi:10.1007/BF00469427

Bradbury SP (1995) Quantitative structure-activity relationships and ecological risk assessment: an overview of predictive aquatic toxicology research. Toxicol Lett 79:229-237. doi:10.1016/03784274(95)03374-T

Bradbury SP, Feijtel TCJ, Van Leeuwen CJ (2004) Meeting the scientific needs of ecological risk assessment in a regulatory context. Environ Sci Technol 38:463A-470A

Brooke LT, Call DJ, Geiger DL, Northcott CE (1984) Acute toxicities of organic chemicals to fathead minnow (Pimephales promelas), vol I. Center for Lake Superior Environmental Studies, University of Wisconsin-Superior, Superior, WI, USA

Escher BI, Hermens JLM (2002) Modes of action in ecotoxicology: their role in body burdens, species sensitivity, QSARs, and mixture effects. Environ Sci Technol 36:4201-4217. doi:10.1021/ es015848h

Geiger DL, Northcott CE, Call DJ, Brooke LT (1985) Acute toxicities of organic chemicals to fathead minnows (Pimephales promelas), vol II. University of Wisconsin-Superior, Superior, Wisconsin, USA

Geiger DL, Poirier SH, Brooke LT, Call DJ (1986) Acute toxicities of organic chemicals to fathead minnow (Pimephales promelas), vol III. Center for Lake Superior Environmental Studies, University of Wisconsin-Superior, Superior, WI, USA

Geiger DL, Call DJ, Brooke LT (1988) Acute toxicities of organic chemicals to fathead minnow (Pimephales promelas), vol IV. Center for Lake Superior Environmental Studies, University of Wisconsin-Superior, Superior, WI, USA

Geiger DL, Brooke LT, Call DJ (1990) Acute toxicities of organic chemicals to fathead minnow (Pimephales promelas), vol V. Center for Lake Superior Environmental Studies, University of Wisconsin-Superior, Superior, WI, USA

Gerritsen A, van der Hoeven N, Pielaat A (1998) The acute toxicity of selected alkylphenols to young and adult Daphnia magna. Ecotoxicol Environ Saf 39:227-232. doi:10.1006/eesa.1997. 1578

Hermens JLM (1990) Electrophiles and acute toxicity to fish. Environ Health Perspect 87:219-225. doi:10.2307/3431028

Jager T, Kooijman SALM (2005) Modeling receptor kinetics in the analysis of survival data for organophosphorus pesticides. Environ Sci Technol 39:8307-8314. doi:10.1021/es050817y

Jager T, Heugens EHW, Kooijman SALM (2006) Making sense of ecotoxicological test results: towards application of processbased models. Ecotoxicology 15:305-314. doi:10.1007/s10646006-0060-x

Jager T, Posthuma L, de Zwart D, van de Meent D (2007) Novel view on predicting acute toxicity: decomposing toxicity data in species vulnerability and chemical potency. Ecotoxicol Environ Saf 67:311-322. doi:10.1016/j.ecoenv.2007.03.003

Kooijman SALM (1996) An alternative for NOEC exists, but the standard model has to be abandoned first. Oikos 75:310-316. doi: $10.2307 / 3546255$

Kooijman SALM, Bedaux JJM, Slob W (1996) No-effect concentration as a basis for ecological risk assessment. Risk Anal 16:445447. doi:10.1111/j.1539-6924.1996.tb01091.x 
Kooijman SALM, Jager T, Kooi BW (2004) The relationship between elimination rates and partition coefficients. Chemosphere 57:745-753. doi:10.1016/j.chemosphere.2004.08.005

Kooijman SALM, Baas J, Bontje D, Broerse M, Jager T, Van Gestel CAM, Van Hattum B (2007) Scaling relationships based on partition coefficients and body sizes have similarities and interactions. SAR QSAR Environ Res 18:315-330. doi: 10.1080/10629360701304196

Meeker WQ, Escobar LA (1995) Teaching about approximate confidence regions based on maximum likelihood estimation. Am Stat 49:48-53. doi:10.2307/2684811

Muenchow G (1986) Ecological use of failure time analysis. Ecology 67:246-250. doi:10.2307/1938524

Newman MC, McCloskey JT (2000) The individual tolerance concept is not the sole explanation for the probit dose-effect model. Environ Toxicol Chem 19:520-526. doi :10.1897/1551-5028 (2000)019<0520:TITCIN $>2.3$. CO; 2

OECD (2006) Current approaches in the statistical analysis of ecotoxicity data: a guidance to application. Organisation for Economic Cooperation and Development (OECD), Paris, France

Russom CL, Bradbury SP, Broderius SJ, Hammermeister DE, Drummond RA (1997) Predicting modes of toxic action from chemical structure: acute toxicity in the fathead minnow (Pimephales promelas). Environ Toxicol Chem 16:948-967. doi :10.1897/1551-5028(1997)016<0948:PMOTAF>2.3.CO;2

Schultz TW, Cronin MTD, Walker JD, Aptula AO (2003) Quantitative structure-activity relationships (QSARs) in toxicology: a historical perspective. Theochem 622:1-22. doi:10.1016/S01661280(02)00614-0

Sijm DTHM, Van der Linde A (1995) Size-dependent bioconcentration kinetics of hydrophobic organic chemicals in fish based on diffusive mass transfer and allometric relationships. Environ Sci Technol 29:2769-2777. doi:10.1021/es00011a011

Spacie A, Hamelink JL (1982) Alternative models for describing the bioconcentration of organics in fish. Environ Toxicol Chem 1:309-320. doi:10.1897/1552-8618(1982)1[309:AMFDTB]2.0. $\mathrm{CO} ; 2$

Sprague JB (1969) Measurement of pollutant toxicity to fish. I. Bioassay methods for acute toxicity. Water Res 3:793-821. doi: 10.1016/0043-1354(69)90050-5

Veith GD, Call DJ, Brooke LT (1983) Structure-toxicity relationships for the fathead minnow, Pimephales promelas: narcotic industrial chemicals. Can J Fish Aquat Sci 40:743-748

Verhaar HJM, De Wolf W, Dyer S, Legierse KCHM, Seinen W, Hermens JLM (1999) An LC50 vs time model for the aquatic toxicity of reactive and receptor-mediated compounds. Consequences for bioconcentration kinetics and risk assessment. Environ Sci Technol 33:758-763. doi:10.1021/es980507y

Zhao Y, Newman MC (2007) The theory underlying dose-response models influences predictions for intermittent exposure. Environ Toxicol Chem 26:543-547. doi:10.1897/06-398R.1 